\title{
Machine Learning Recognition of Gait Identity via Shoe Embedded Accelerometer
}

\author{
Silvia Strada ${ }^{1, *}$, Jacopo Paris ${ }^{1, *}$, Fabio Piccoli ${ }^{1}$, Davide Pietro Tucci ${ }^{1}$, Patrizia Casali ${ }^{2}$, and Sergio Savaresi ${ }^{1}$
}

\begin{abstract}
Machine learning and data analytics are becoming pervasive also in the analysis of human behaviour as more and more miniaturized sensors can observe and quantify human activities. The so-called wearables are particularly tiny transducers which give the possibility to understand an individual's behaviour possibly enabling innovative services such as gait analysis based identification, foot pressure analysis, fall prevention and automatic recognition of dangerous situations. In this work we investigate an innovative experimental setting where accelerations are captured, during walking, by an Inertial Measurement Unit embedded in the shoe's sole. The goal is to identify who is wearing the shoe by simply analyzing his gait. User classification is then performed comparing different machine learning methods, relying either on the $k$-NeareastNeighbour or on the Linear Discriminant Analysis algorithm. An extensive experimental campaign was carried out on five young adults and a comparative analysis of the accuracy of the methods proves that machine learning recognition of gait identity via shoe embedded accelerometer is feasible and sufficiently reliable.
\end{abstract}

\section{INTRODUCTION AND PROBLEM STATEMENT}

New opportunities in the market, such as the internet of things (IoT) and smart mobile devices, have accelerated the development of wearable technology. Wearables ensure many benefits to users, offering them a sense of safety and productivity while providing health incentives or longterm and continuous monitoring of physiological parameters. Provided that wearable devices can also automatically define the identity of a currently monitored person, the respective measurement data would be very useful to activate some assistance strategy or to intervene with implementation tools, if available.

In particular, gait analysis has benefited from the advent of wearables, making this research area quite attractive. The personal way of walking carries a series of important information that can be exploited both for medical applications, such as pathologies diagnosis [1], [2], which is not the issue tackled in this paper, and for security purposes, such as

\footnotetext{
${ }^{1}$ Silvia Strada, Jacopo Paris, Fabio Piccoli, Davide Pietro Tucci and Sergio Savaresi are with the Dipartimento di Elettronica, Informazione e Bioingegneria, Politecnico di Milano, Piazza Leonardo da Vinci 32, 20133 Milan, Italy. Email: \{silvia.strada | sergio.savaresi\}epolimi.it, \{jacopo.paris | fabiol.piccoli | davidepietro.tucci\}@mail.polimi.it

${ }^{2}$ Patrizia Casali is with E-Novia, Via San Martino 12, Milan 20122, Italy. Email: patrizia.casalide-novia.it

* corresponding author
}

biometric identification [3], [4], [5]. Moreover, gait recognition based on such devices doesn't need an explicit user interaction. Gait-based biometric studies can be classified into three categories: machine vision-based, [6], wearable sensor-based and floor sensor-based ones, [7]. This work falls in the ambiance of wearable inertial sensor-based approaches and, as such, has the advantage of not requiring sophisticated specialized equipment such as high definition cameras or pressure/force sensing ground-platforms.



Fig. 1: The Wahu shoe with its innovative active sole.

Many studies have proved that human gait is a unique feature that can be used for the robust identity recognition, for example, [8], [9]. Among the numerous research works on gait-based biometrics, in [10] authors suggest a smartphonebased gait recognition method built on data collected from young adults walks and on machine learning, while gait recognition is performed in [11] with different methods. Instead, Gadaleta et al. proposed an approach that relies on convolutional neural networks [12]. The feasibility of gait recognition was demonstrated in these studies for young adults, where the gait fluctuation is relative small since they have a quite constant and established walking style and a stable gait pattern.

This paper deals with two machine learning, gait-based, identity recognition solutions whose innovativeness lies in the fact that the sensed accelerations are collected through an Inertial Measurement Unit (IMU) placed and glued into the sole of the shoe. The final aim is to embed the IMU in the new Wahu shoe, Fig 1, both for sensing purposes and then for actuation. There will be the possibility of creating innovative services for the user, such as gait analysis based identification, foot pressure analysis or fall prevention. The ultimate goal of this active sole will be to adapt its morphology both to the external environment, like terrain, 
temperature or humidity, and to the state of the user.

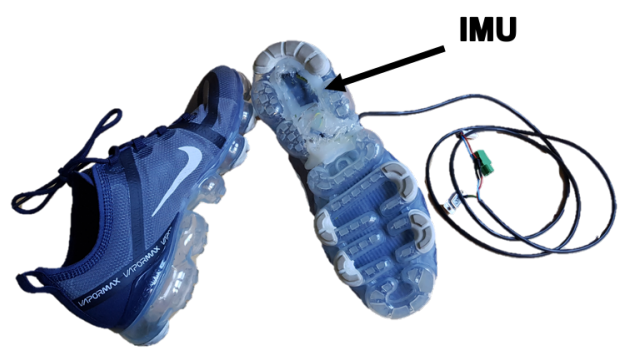

Fig. 2: The sneaker used for the test campaign with the IMU embedded and glued in the right-shoe heel.

\section{EXPERIMENTAL SETUP}

Since one of the main criticalities related to the identity detection problem via worn sensors is the repeatability of the experiments, the sensors themselves must be the most permanently attached to the individual's walk. Several alternative solutions would have been possible, as already analyzed in the work of Derawi, [13], in particular it is common to equip with sensors ankles, hips and legs. In our work it was decided from the beginning to embed the sensor elements in the shoe. Indeed, this decision is mainly motivated by the application itself, since the final product will be the Wahu sensorized shoe. In particular, the employed sensor is the IMU LSM6DS3HTR, whose specifications (storage capacity, self-life, battery usage, etc.) can be found in [14]. For ensuring that each experiment is done with exactly the same reference system, the IMU is fixed on a tiny board, powered by a $8.4 \mathrm{~V}$ external battery, under the heel of a common sneaker shoe employed for the test campaign. The IMU is drowned in the sole with hot glue, which works also as a shield from possible damages (see Fig. 2), and connected by-wire, through a Vector CANalyzer data logger, to a laptop computer. The IMU firmware is written in order to collect the accelerations along the three axes defined as in Fig. 3. with a sampling frequency of $200 \mathrm{~Hz}$. Data from the gyroscope, also collected by the IMU, are measured but not stored for now, leaving the employment of angular velocities information to a possible future development of the research.

On the basis of the above described data acquisition equipment, the routine of the experiments is defined and, in particular, each tester is asked to walk straight on a flat pavement as much as possible maintaining a constant speed. The trials have been carried out on different types of surfaces, assuming the terrain flat and without a relevant roughness. Specifically, the tester had to walk repeatedly indoor on linoleum floor and outdoor on an asphalt road.

\section{DATA PRE-PROCESSING}

As previously illustrated, the available walking data are captured from a 3-axis accelerometer and are denoted as

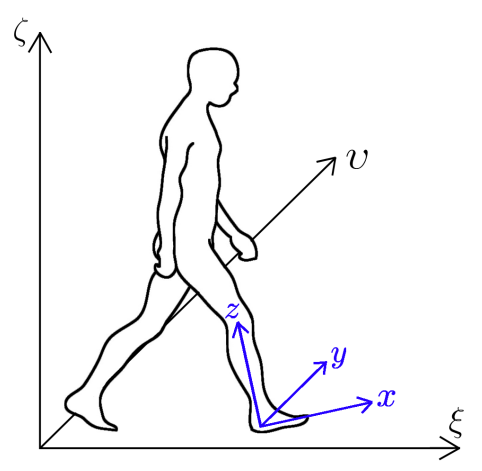

Fig. 3: The orientation of the IMU reference system $(x, y, z)$ with respect to the user one $(\xi, v, \zeta)$.

$a_{x}, a_{y}$ and $a_{z}$. The three accelerations are not treated separately, instead a combined signal was used, simply consisting of the sum of the three accelerations:

$$
a_{c}=a_{x}+a_{y}+a_{z}
$$

Other combinations of the three elementary signals were tested but provided much weaker results. The choice of compacting the three accelerations is a common practice in many wearable related algorithms, see for example [15], and it is justified by two main considerations:

- using a single compact signal makes the algorithm more efficient and this aspect is crucial in view of our future on-board implementation;

- exploiting the total acceleration signal allows a better identification of the gait typical peaks and valleys, easing the job of segmenting the overall walk into many subsequent steps.

Once the total acceleration signal, $a_{c}$, is computed, our approach consists of primarily decomposing the whole gait into a sequence of single steps; a velocity filter removes potential steps outside the nominal range of velocities considered as acceptable. The individual steps are then normalized over a fixed number of samples and finally fed to a machine learning (ML) algorithm capable of predicting the user whom the steps belong to. The overall procedure, shown in Fig 4, will be analysed in the following section. MATLAB has been used for the algorithm implementation.

The filtering phase is composed of two different and parallel smoothing operations:

- a low-pass filter with bandwidth $0.01 \mathrm{~Hz}$ returns the signal employed for valleys detection. Due to this operation, a considerable amount of information about the gait are lost, but the obtained noise-filtered wave still preserves the real position of its stationary points, which are much better discernible;

- a low-pass filter with bandwidth $10 \mathrm{~Hz}$ returns the signal that will be actually used in the classification stage. The higher cut-off frequency allows to attain a signal still able to univocally describe the user's gait 


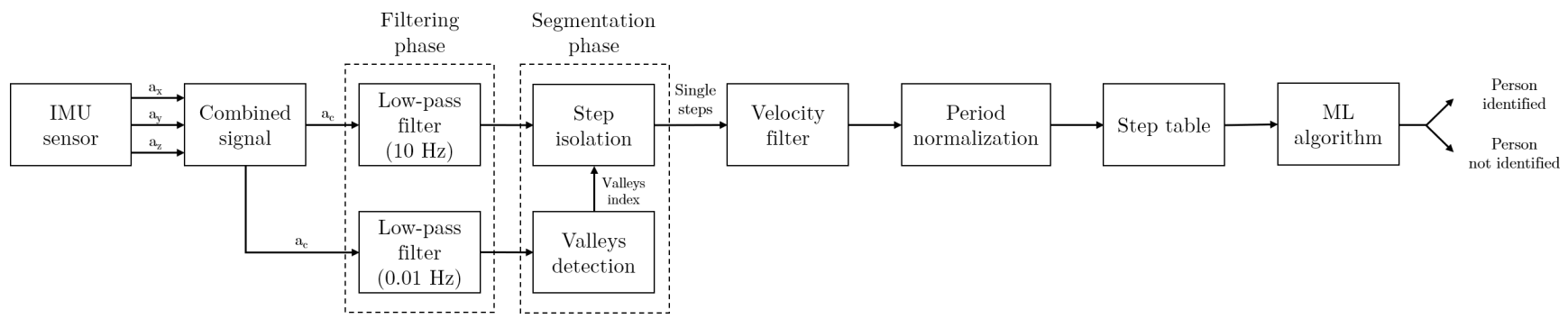

Fig. 4: The workflow chart of the algorithm from the sensor raw data to the classification output label. The block diagram shows all the intermediate stages through which the signals flow, before being fed to the machine learning algorithm.

signature, removing at the same time the high-frequency measurement noise.

In the segmentation phase a negative-peaks, i.e valleys, detection algorithm is implemented to identify and successively isolate the single steps within a gait. This particular choice is simply justified stressing the behaviour of signal $a_{c}$, shown in Fig 5. Indeed, the presence of two maxima in each step i may induce MATLAB to a wrong decomposition of the entire walk, while, on the contrary, the detection of the valleys negative minima, clearly distinguished one from the other, allows an identification less incline to potential errors. To perform this task, the MATLAB built-in function findpeaks is exploited and its optional parameters Minimum Peak Distance and Minimum Peak Height are suitably tuned; in particular, these two empirical thresholds are fixed in order to specify the minimum distance between two adjacent valleys and their minimum depth. As a result, the output of the valleys detection are the integer indices of the samples characterizing the valleys in the signal $a_{c}$. These information are used in the following to split the entire gait acceleration into single steps.

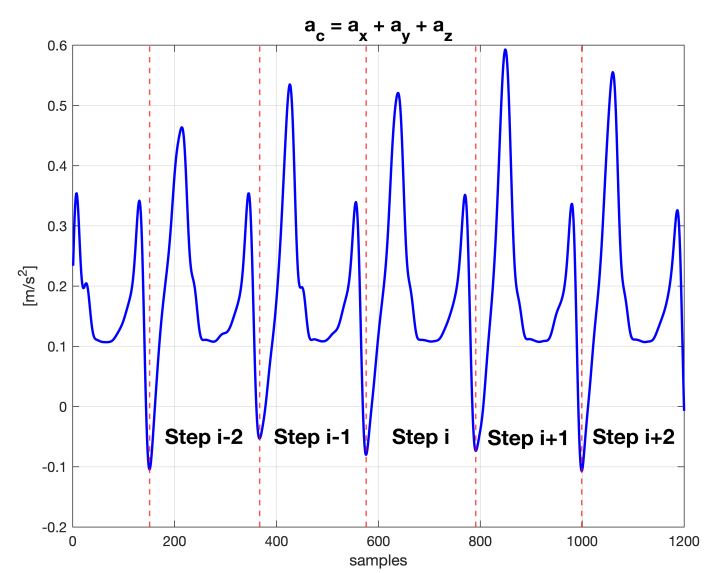

Fig. 5: The waveform of $a_{c}$ smoothed with the low-pass filter at $0.01 \mathrm{~Hz}$. The vertical red dashed lines locate the boundaries of each single step.

A velocity filter is next implemented with the aim of removing the steps that are too slow or too fast within the gait. In order to establish lower and upper bounds $\left(\mathrm{N}_{\text {samples }}^{\text {min }}\right.$, $\mathrm{N}_{\text {samples }}^{\max }$ ) on the total number of samples that we decide to associate to the description of a single "standard velocity" step, an additional series of tests was carried out with participants walking very slowly or at a higher speed. All steps characterized by a number of samples smaller than $\mathbf{N}_{\text {samples }}^{\text {min }}$ or greater than $\mathrm{N}_{\text {samples }}^{\max }$ are eventually discharged. This velocity filter also provides a serviceable tool to ultimately remove wrong or outlier steps obtained as output of the segmentation phase.

At this point, all the single available steps for each tester's walk are considered "valid", but they still are characterized by a generic number of samples belonging to the interval $\left[\mathrm{N}_{\text {samples }}^{\text {min }} ; \mathrm{N}_{\text {samples }}^{\max }\right]$. To make all steps directly comparable with each-other, a step-length normalization is performed using MATLAB interpl function, which employees the nearest neighbour interpolation method. The period length was set to 210 samples, that is the average length of a step when accelerometer's data are sampled at our frequency of $200 \mathrm{~Hz}$.

The final product of the data pre-processing phase is the step table which stores the time-amplitude information of each user's step, i.e. a table with as many rows as the overall number of steps, labelled for each user, and as many columns, 210, as the number of samples that describe a "standard velocity" young adult step, post-processed with a period normalization.

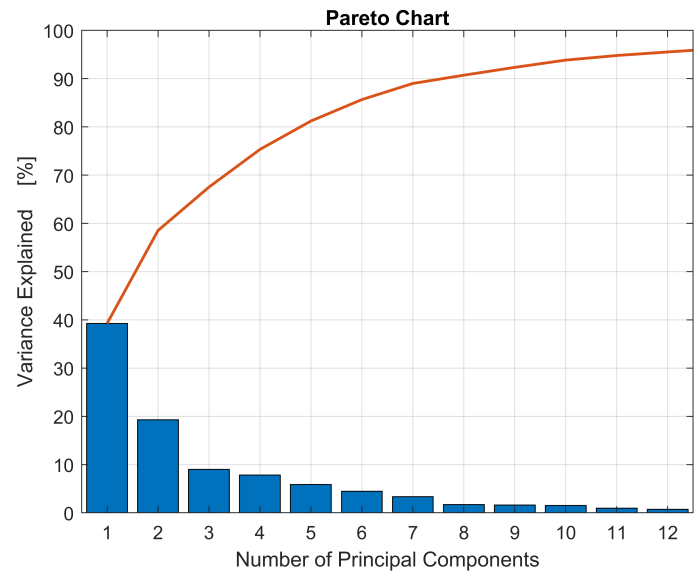

Fig. 6: The Pareto Chart shows the trade-off between the variance explained by the signal after the dimensionality reduction and the consequent number of PCs taken. 


\section{MACHINE LEARNING ALGORITHMS FOR IDENTITY RECOGNITION}

In this Section we illustrate the two methods aimed at identifying the individual who is wearing that certain shoe by means of his gait signature expressed via acceleration data collected during normal walking experiments from the IMU, integral with the shoe's sole. The first relies on a standard algorithm, the k-Nearest-Neighbour [16], which does not require any a-priori assumption on the collected data, the second, the Linear Discriminant Analysis [17], is based on the hypothesis that the observations in each prediction class can be modelled with a normal probability distribution. Overall, the classification procedure establishes a mapping between the pre-processed acceleration signal and the person to be recognized.

Before entering in the details, a preliminary consideration, valid for both the methods, should be made. A first idea was to pick the predictors to be fed to the ML algorithms, among some standard statistical features like mean, variance, skewness index and kurtosis index, quantities easy to be associated to characteristics of the signal $a_{c}$. As long as the classification algorithm was tried out on a reduced subset of the overall dataset, the statistical predictors provided satisfactory results in terms of accuracy, however performance downgraded using the whole bunch of data.

Therefore, the innovative intuition of this paper is to select as features directly the samples of each step for a total of 210 predictors per step. It is evident that a so accurate description of the signal is more keen to univocally discriminate one walk from another, however the dimensionality of such a problem is difficult to be managed. To overcome this drawback, the Principal Component Analysis (PCA) is employed, see [18].

The minimum variance in the data that has to be explained by the PCs is a user choice, since obviously the higher the number of the PCs included, the smaller the loss of information. In our setup, this threshold is fixed at $95 \%$, see Fig 6 leading to the employment of 12 PCs. The goodness of this compromise is illustrated in Fig 7. Overall, the PCA approach can be easily interpreted, from a practical point of view, as a method that allows to describe (loosing just $5 \%$ of data variability) each single step with at most 12 out of 210 integer numbers.

A final remark concerning the combined signal introduced in Section III should be made. Fig 8 gives a preview of the performance in terms of classification error provided by one of the two ML algorithms, will will in depth discussed in Section IV-A and Section V. The aspect we want to emphasize here, is that the PCA validates how the information carried by $a_{c}$ is actually the most performing one, compared to the single accelerations $a_{x}$ or $a_{z}$.

\section{A. k-Nearest-Neighbours (k-NN) and Linear Discriminant Analysis (LDA) algorithms}

The well-known $k$-Nearest-Neighbour algorithm, [16], is a machine learning, non-parametric method used for classification and regression. It relies on the concept of distance
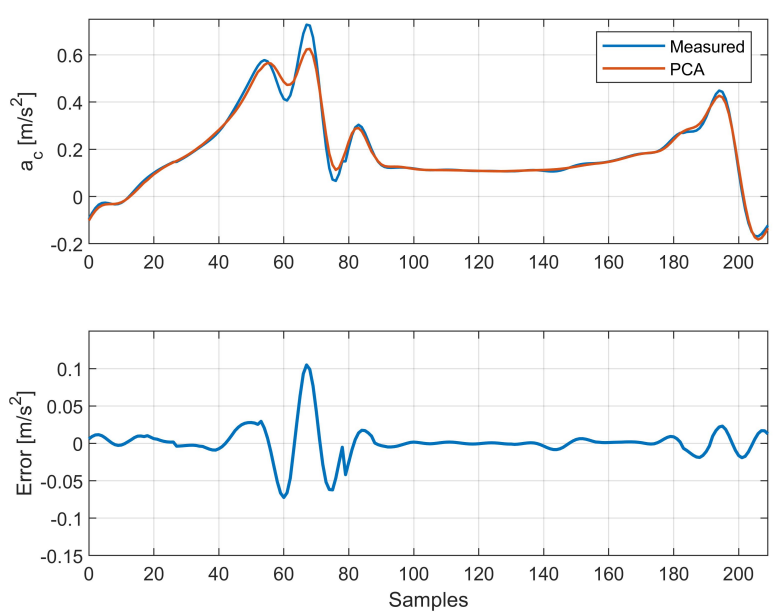

(a)


(b)

Fig. 7: The upper plot in (a) and (b) represents the superimposition of the real measured acceleration signal (blue) and the PCA-reconstructed one (orange), the lower plot represents the error between the two signals. In (a) only the first PC is considered, while in (b) 12 PCs.

between data points. In this work, the Euclidean norm is employed as the distance metric because it has exhibited the best performance compared to other norms.

A data item is classified by a majority vote of its neighbours, with the object being assigned to the class most common among its $k$ nearest neighbours. The value of the integer $k$, usually kept small, is a critical point in the tuning of the algorithm, since a weakness of the $k$-NN is indeed that it is particularly sensitive to the local structure of the data. Therefore, in our experiments, an accurate sensitivity analysis was carried out and it resulted that the optimal choice was $k=3$.

The second proposed ML approach is the Discriminant Analysis. This algorithm uses the training observations to determine a boundary between the response classes, which 




Fig. 8: The classification error trend as function of the number of PCs included. The dynamics are attained considering the combined signal $a_{c}$ or the standard ones $a_{x}$ and $a_{z}$.

is determined by treating the observations of each class as samples from a multi-dimensional normal distribution. Then, observations on one side of the boundary would be classified as belonging to one class and the others to the other class. The geometrical type of the boundaries (linear, quadratic, etc...) implies different classification procedures: in this work, we assume that all the distributions have the same shape, i.e. described by the same covariance matrices, so that the boundaries turn out to be linear. In particular, the Linear Discriminant Analysis, [17], results quite easy to perform, even with a small memory overhead. The performance of both classifiers are discussed in Section $\mathrm{V}$.

\section{B. High-level techniques for an exact classification}

The novelty of this paper is to introduce two advanced methods which rely on the output of the algorithms just described in Section IV-A, but allow to obtain better and accurate classification results. The underlying idea is that recognizing a person by analyzing only one single step extracted from his gait is clearly an interesting task from a theoretical point of view, but not strictly required in practice. Instead, asking the user to make a short walk would be a reasonable request and in turn, it would lead to a more precise and correct identification.

In the first proposed approach, the classification is thus no more performed on a single step, but on a mean step, which is obtained by picking and averaging $m$ consecutive steps. Consequently, a sensitivity analysis on the optimal value of the parameter $m$ was accomplished and as $m$ increases, performance improved as well. The intuitive reason is that the information carried by a mean step is able to characterize in a better and precise way a person's walking pattern. The main drawback of this technique is that if one step is clearly wrong, e.g. it corresponds to a stumble, also the resulting mean step will be affected, leading to a potential misclassification.

To cope with this problem and hence make the classification robust with respect to potential outliers, another methodology has been investigated. The insight is to require the user to perform again $m$ consecutive steps, defining a so called folder, but now without averaging them. For each step belonging to the folder, the identification algorithm, i.e. the $k$-NN or the LDA, will be run. An empirical threshold $T$ has to be fixed, in our setup $T=50 \%$. If more than $T$ [\%] steps in the folder are identified as belonging to the same person, then the identification procedure can be considered successful, otherwise another set of $m$ steps should be repeated until the recognition procedure turns out to be successful (see the example in Fig 9 ).

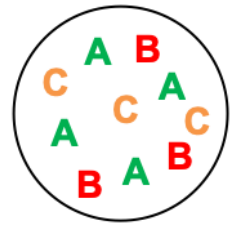

No person identified

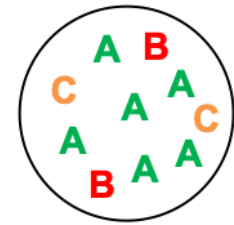

Person A identified
Fig. 9: Example of the folder version high-level technique for an exact classification with $m=10$ and $T=50 \%$

\section{Test CAMPAign AND Discussion OF THE EXPERIMENTAL RESULTS}

As already highlighted in Section III, the experiments were carried out by 5,23 years old, male subjects in good health and physical conditions. From the tests, we collected $12-15$ minutes of walk for each tester, with an overall of around 3700 steps. All these data have been divided into a training set and a validation set, in particular 1932 steps were used to train the machine learning algorithm and the remaining 1802 were used for the validation. This partition was done in order to obtain two balanced clusters of data.

So, running the $k$-NN algorithm with this setup we obtain an accuracy in the identity identification equal to $97.8 \%$ which means that 1763 steps are classified correctly while 39 steps are associated to the wrong subject. In addition, running the LDA algorithm with the same setup we obtain an accuracy equal to the $99 \%$, Fig 10 , which means that 1784 steps are classified correctly while only 18 steps are associated to the wrong subject. These results prove that the proposed identity recognition methodology based on quite simple machine learning algorithms is not only feasible, but also very accurate. A comparison of the proposed approach with other different ones found in literature is reported in Tab. I

\begin{tabular}{lccc}
\hline DEVICE \& POSITIONING & ALGORITHM & ACCURACY & REF. \\
\hline Smartphone in pocket & CNN & $94 \%$ & {$[12]$} \\
Accelerometer on ankle & t-test & $91 \%$ & {$[15]$} \\
MMR on calf & SVM & $98 \%$ & {$[19]$} \\
Accelerometer on belt & DTW & $94.3 \%$ & {$[20]$} \\
Accelerometer under heel & LDA & $99 \%$ & Our \\
\hline
\end{tabular}

TABLE I: The performance of sensor-based gait recognition methods. 


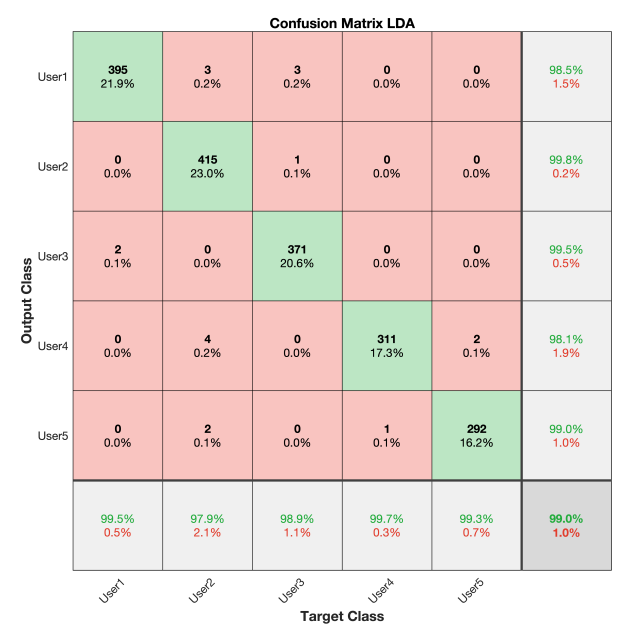

Fig. 10: The Confusion Matrix related to the LDA algorithm.

In order to further reduce the classification error, the two high level techniques for an exact classification introduced in Section IV-B can be applied. The first one, guarantees a convergence to $100 \%$ accuracy using at least $m=10$ steps; the second one guarantees the convergence to $100 \%$ accuracy using a folder made up of at least of $m=6$ steps, which in both cases is a not too tiresome request for the tester. A summary of the achieved performance with the different methods is illustrated in Tab. II.

\begin{tabular}{lr}
\hline Algorithm & Accuracy [\%] \\
\hline$k$-NN & $97.8 \%$ \\
LDA & $99 \%$ \\
\hline Mean-step version $(m \geq 10)$ & $100 \%$ \\
Folder version $(m \geq 6)$ & $100 \%$ \\
\hline
\end{tabular}

TABLE II: Classification performance results.

\section{CONCLUSION AND FUTURE WORK}

In this paper, an innovative approach is proposed for gait identity recognition based on inertial data collected by an IMU glued in the shoe's sole, then processed by two different machine learning algorithms. The accurate classification of the individual wearing the sensorized shoes is accomplished by employing the informative content of the 3 -axis accelerometer measurements, a remarkable novelty in the state of the art of wearable-based human gait recognition. The proposed algorithms were trained and validated on an initial, starting dataset of five young adults' walking patterns, because, in order to demonstrate the feasibility of the idea, the learning procedure relied on a fairly primitive experimental apparatus. It will shortly be made much more robust in order to allow the repeatability of much more numerous experimental training, validation and testing steps. Within these forthcoming experiments, also more heterogeneous subjects will be taken into account, in order to globally authenticate the effectiveness of the presented approaches. Moreover, further planned phases of this research work will be:
- upgrading of the algorithms in order to entail also the presence of curvilinear trajectories, thus giving to the individual the chance to be identified not only through a straight walking pattern;

- implementing an automatic detection and removal of walking steps which are clearly faulty;

- investigating the possible added value of the gyroscope's measurements.

\section{REFERENCES}

[1] S. Chen, J. Lach, B. Lo, and G. Yang, "Toward pervasive gait analysis with wearable sensors: A systematic review," IEEE Journal of Biomedical and Health Informatics, 2016.

[2] C. Ricciardi, M. Amboni, C. De Santis, G. Improta, G. Volpe, L. Iuppariello, G. Ricciardelli, G. D'Addio, C. Vitale, P. Barone, et al., "Using gait analysis' parameters to classify parkinsonism: A data mining approach," Computer methods and programs in biomedicine, vol. 180, p. 105033, 2019.

[3] S. Sarkar, P. J. Phillips, Z. Liu, I. R. Vega, P. Grother, and K. W. Bowyer, "The humanid gait challenge problem: data sets, performance, and analysis," IEEE Transactions on Pattern Analysis and Machine Intelligence, vol. 27, no. 2, pp. 162-177, 2005.

[4] N. Al-Naffakh, N. Clarke, F. Li, and P. Haskell-Dowland, "Unobtrusive gait recognition using smartwatches," in 2017 International Conference of the Biometrics Special Interest Group (BIOSIG), 2017.

[5] J. Moon, N. H. Minaya, N. A. Le, H.-C. Park, and S.-I. Choi, "Can ensemble deep learning identify people by their gait using data collected from multi-modal sensors in their insole?," Sensors, 2020.

[6] A. Mosenia, S. Sur-Kolay, A. Raghunathan, and N. Jha, "Caba: continuous authentication based on bioaura," IEEE Trans. Comput., vol. 66 (5), pp. 759-772, 2017.

[7] H. Ma and W. Liao, "Human gait modeling and analysis using a semimarkov process with ground reaction forces," IEEE Trans. Neural Syst. Rehabil. Eng., vol. 25 (6), p. 597-607, 2017.

[8] S. Sprager and M. J. , "Inertial sensor-based gait recognition: a review,"Sensors, vol. 15 (9), p. 22089-22127, 2015.

[9] N. Bora, G. Molke, and H. Munot, "Understanding human gait: A survey of traits for biometrics and biomedical applications," in Proceedings of the International Conference on Energy Systems and Applications, pp. 723-728, 2015.

[10] M. Muaaz and R. Mayrhofer, "Orientation-independent cell phone based gait authentication," in Proc. 12th Int. Conf. Advances in Mobile Computing and Multimedia, pp. 161-164, 2014.

[11] T. Ngo, Y. Makihara, and H. Nagahara, "Performance evaluation of gait recognition using the largest inertial sensor-based gait database," in Proceedings of the 5th IEEE IAPR Int. Conf. on Biometrics (ICB), pp. 360-366, 2012.

[12] M. Gadaleta and M. Rossi, "Idnet: Smartphone-based gait recognition with convolutional neural networks," Pattern Recognition, 2018.

[13] M. Derawi, "Accelerometer-based gait analysis, a survey," Nor Informasjonssikkerhetskonferanse NISK, 012010.

[14] STMicroelectronics, "Imu lsm6ds3htr," 2017.

[15] D. Gafurov, K. Helkala, and T. Sondrol, "Biometric gait authentication using accelerometer sensor," J. Comput, vol. 1 (7), pp. 51-59, 2006.

[16] J. Novakovic, A. Veljovic, S. Ilic, and M. Papic, "Experimental study of using the k-nearest neighbour classifier with filter methods," in Proceedings of the Conference on Computer Science and Technology, pp. 91-99, 2016.

[17] "Machine learning with matlab - a self paced course," 2019. Mathworks.

[18] H. Abdi and L. J. Williams, "Principal component analysis," WIREs Computational Statistics, 2010.

[19] O. Feshier, Y. Nafea, A. Hesham, and W. Gomaa, "Gait-based person identification using multiple inertial sensors," 072020.

[20] M. O. Derawi, P. Bours, and K. Holien, "Improved cycle detection for accelerometer based gait authentication," in 2010 Sixth International Conference on Intelligent Information Hiding and Multimedia Signal Processing, pp. 312-317, IEEE, 2010. 